\title{
Do we need to overcome barriers to learning in the workplace for foundation trainees rotating in neurosurgery in order to improve training satisfaction?
}

\author{
This article was published in the following Dove Press journal: \\ Advances in Medical Education and Practice \\ 30 March 2016 \\ Number of times this article has been viewed
}

\author{
Pho NH Phan' \\ Keyur Patel ${ }^{2}$ \\ Amar Bhavsar ${ }^{3}$ \\ Vikas Acharya ${ }^{4}$ \\ 'Warwick Medical School, University \\ of Warwick, Coventry, ${ }^{2}$ Luton and \\ Dunstable University Hospital, Luton, \\ ${ }^{3}$ Department of Neurosurgery, Queen \\ Elizabeth Hospital, Birmingham, \\ ${ }^{4}$ Department of Neurosurgery, \\ University Hospitals Coventry and \\ Warwickshire, Coventry, UK
}

\begin{abstract}
Junior doctors go through a challenging transition upon qualification; this repeats every time they start a rotation in a new department. Foundation level doctors (first 2 years postqualification) in neurosurgery are often new to the specialty and face various challenges that may result in significant workplace dissatisfaction. The neurosurgical environment is a clinically demanding area with a high volume of unwell patients and frequent emergencies - this poses various barriers to learning in the workplace for junior doctors. We identify a number of key barriers and review ideas that can be trialed in the department to overcome them. Through an evaluation of current suggestions in the literature, we propose that learning opportunities need to be made explicit to junior doctors in order to encourage them to participate as a member of the team. We consider ideas for adjustments to the induction program and the postgraduate medical curriculum to shift the focus from medical knowledge to improving confidence and clinical skills in newly qualified doctors. Despite being a powerful window for opportunistic learning, the daily ward round is unfortunately not maximized and needs to be more learner focused while maintaining efficiency and time consumption. Finally, we put forward the idea of an open forum where trainees can talk about their learning experiences, identify subjective barriers, and suggest solutions to senior doctors. This would be achieved through departmental faculty development. These interventions are presented within the context of the neurosurgical ward; however, they are transferable and can be adapted in other specialties and departments.
\end{abstract}

Keywords: medical education, foundation program, junior doctors, induction program, neurosurgery, learning in the workplace

\section{Introduction}

Neurosurgery is a niche specialty in the UK and has its own run-through training program with specialist teaching/training for their own trainees, rather than being part of a generic national/regional surgical training program. In this specialty, specifically, the clinician is a surgeon as well as a physician who looks after their own postoperative and medical complications, which may or may not be related to their primary neurosurgical pathology.

The neurosurgical ward is a busy and challenging environment for junior doctors. Here, frequent clinical emergencies and service demands commonly act as barriers to learning. According to foundation trainees, intense workloads, large volumes of patients, regular medical emergencies, and increasing administrative tasks make it difficult to have a positive learning experience or actually allocate time for learning/
Correspondence: Vikas Acharya

Department of Neurosurgery,

University Hospitals Coventry and

Warwickshire, Clifford Bridge Road,

Coventry CV2 2DX, UK

Tel +442476964000

Email vikas.acharya@doctors.org.uk
Advances in Medical Education and Practice 2016:7 21।-2/7

2 II

Dovepress

http://dx.doi.org/10.2147/AMEP.S96028 (c) (1) (5) 2016 Phan et al. This work is published and licensed by Dove Medical Press Limited. The full terms of this license are available at https://www.dovepress.com/terms.php
and incorporate the Creative Commons Attribution - Non Commercial (unported, v3.0) License (http://creativecommons.org/licenses/by-nd/3.0/). By accessing the work you

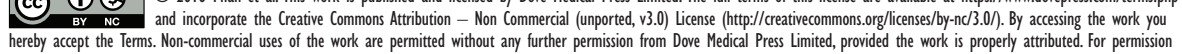

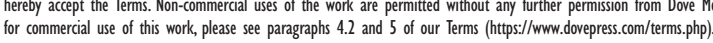


teaching. In addition, as the trainees' daily workload varies by the hour, the ward cannot and does not always offer the same learning opportunities to all learners. ${ }^{1}$

A number of key barriers to learning for foundation trainees in the workplace have been identified: feeling underprepared for their jobs, perception of the workplace and integration into the team, not having a clear and structured curriculum, and generally not receiving enough teaching from senior clinicians due to the demanding clinical environment. For the purposes of ease of identification and comparability, foundation doctors/trainees will be generically replaced with junior doctors.

This article reviews the literature on these important barriers to junior doctors' training in general, and in neurosurgery specifically. Subsequently, we put forward a number of proposals on relevant interventions that could be trialed in the clinical workplace to improve the learning experience and satisfaction of foundation trainees.

\section{Barriers to learning in the workplace Lack of confidence in newly qualified doctors}

It is important to acknowledge the psychological changes from medical student to junior doctor. The consensus is that most doctors find the transition abrupt and their new jobs stressful. ${ }^{2}$ Health care professionals across a variety of medical specialties agree that, upon qualification, they generally feel underprepared for their first job. ${ }^{2-4}$ Before starting their training, junior doctors commonly experience negative perception of their own abilities. In addition, their confidence and clinical skills are both found to be lacking upon assessment during the induction period. ${ }^{5,6}$

Evidence shows that a common problem for new trainees is the lack of formal guidance and support from colleagues, ${ }^{7}$ which leaves many new doctors feeling redundant. This is intensified through a general lack of recognition and the repetitive nature of some daily jobs.

\section{Theory-based induction program}

In 2008, an induction program was developed and evaluated for newly qualified doctors entering foundation practice in the UK. All the trainers surveyed felt that it is important to have a standardized program, and junior doctors were of the opinion that the current program, being all lecture based, does not add much value to what they already knew, and therefore, does not influence their daily work. This has been again found in the literatures concluding that, before commencing their jobs, junior doctors often lack confidence and ability with regard to clinical skills. ${ }^{5,6}$ Shadowing and clinical skills' training is further advocated in the literatures, and specifically encourages full days of shadowing to allow the transmission of experience and skills from the outgoing doctors. ${ }^{5}$ This is prudent to be highlighted as many neurosurgical units unfortunately do not have a standardized induction program; this can have a detrimental impact on foundation doctors' readiness and confidence before starting the work. ${ }^{8}$

\section{Junior doctors' perception of the workplace}

Another barrier to learning in the workplace is unevenly distributed workplace affordances ${ }^{9}$ or inadequate assignment of duty at work. Generally, junior doctors thrive when given responsibility. This makes them feel more integrated in the team, and their role is worthwhile. It also gives them ownership over patient care and satisfies their desire to treat. On the contrary, when not delegated enough responsibility, self-esteem, and consequently, motivation to learn is low among junior doctors, as they perceive their job as only involving daily clerical and simple clinical tasks. As a whole, junior doctors' feelings about morale, enjoyment, and job satisfaction seem to vary depending upon their rotations, colleagues, learning experiences, and how welcomed they feel in the team. ${ }^{10}$

\section{Are ward rounds too busy for teaching/ learning?}

Conversely, the busy clinical workload and medical emergencies should increase opportunistic learning. In principle, junior doctors want to learn and senior clinicians want to teach, and therefore, a busy department means that there are ample opportunities for learning/teaching to take place. However, in reality, it unfortunately does not. A prime example of this is the daily ward round. When surveyed, senior clinicians feel that the ward round is a good way of providing opportunistic and relevant teaching but share concerns about the increase in time needed to complete the round. Consequently, they often do not take time during rounds to teach, while junior doctors feel too pressurized by the workload to ask for teaching or even questions related to the clinical work. As a result, up to $70 \%$ of trainees disagree or are uncertain that they learn something new from the ward round every day, ${ }^{11}$ as they are more preoccupied with the administrative tasks and/or being vigilant of their bleeps going off, and eventually lose interest in what they can learn from the patients.

Even when teaching/learning does occur during ward rounds, it has been observed that there is frequently some 
discrepancy between the teaching styles favored by senior doctors and the learning styles of trainees. Consultants are of the opinion that they should role model certain skills, attitudes, and behaviors, instead of imparting medical information. On the other hand, junior doctors would rather learn clinical knowledge. This subsequently creates a gap in understanding between the teacher and the learner, and the educational value of the ward round is, therefore, not maximized.

\section{Generic medical curriculum}

Finally, we need to address the need to reform the current curriculum in postgraduate medical education, ${ }^{12-14}$ especially in relation to junior doctors in neurosurgery. While all junior doctors in training have a generic curriculum to follow, this is unlikely to be applicable or sufficient in neurosurgery due to its specialist nature. Their current curriculum does not correlate with the early years neurosurgical curriculum learning objectives and actual daily work, the onus is on the juniors to carry out self-directed study in their own time to make up for this. However, they do not always have the spare time for this. Senior clinicians to feel that they cannot provide teaching on focused or specialist topics, as these trainees do not have the sufficient basic knowledge and prior preparation in order to benefit/apply the detailed knowledge. Either the foundation program (junior doctor) curriculum needs to incorporate neurosurgery into its training/teaching or at least basic teaching needs to be provided specifically for those rotating in neurosurgery.

\section{Proposed interventions to improve junior doctors' learning experience}

There are several reasons why at present the trainees' learning does not always rise to the forefront in a neurosurgical department. These barriers include an extremely busy working environment with time limitations, frequent clinical emergencies, complex patient presentations, fatigue of the supervising/senior clinician, and ongoing service pressure requiring acute service provision as opposed to teaching and training.

There are several different methods or interventions to attempt to improve the satisfaction for foundation trainees rotating through neurosurgery, many of which have already been tried elsewhere. We propose those considered within our neurosurgical department following a successful trial by one of the authors in the department of obstetrics and gynecology in a different hospital. Following discussions with trainees and trainers, these specific interventions were deemed likely to improve trainee satisfaction within the specialty of neurosurgery.

\section{Clinically relevant induction program}

To reform the notion that a junior doctor's immediate postqualification training is one to be endured and not enjoyed and to encourage learning during this period, the workplace environment should be one where learning objectives are clearly structured and facilitated from the outset. ${ }^{7}$ This should start from the time of induction to the department. Rather than a fixed schedule of lectures, the content of the induction program should be more clinically relevant to the specialty in which they are rotating through, and emphasis should be on giving junior doctors the necessary guidance to make an effective start. They should be able to confidently carry out appropriate examinations and skills/procedures and make clinical decisions based on their findings. Juniors should also have the opportunity to reflect upon and articulate their formative learning experience openly. A potential solution is to provide ongoing systematic training for both trainees and trainers, rather than a fixed period at the beginning of the rotation(s).

A survey was carried out locally within the Department of Neurosurgery at University Hospitals Coventry and Warwickshire with the aim of gaining information on the junior doctors rotating in neurosurgery relating to their experience and thoughts on their induction program. It was highlighted that the program did not give them the opportunity to learn, develop, or practice relevant clinical skills, despite neurosurgery being a very skill-based specialty. Subsequently, an intervention was trialed, this included practical sessions on sampling cerebrospinal fluid from external ventricular drains, performing lumbar punctures and removing wound drains in addition to clinically relevant talks on managing neurosurgical patients on the ward and the commonest complications. This intervention received very positive feedback from both the junior and senior doctors in the department. Throughout, a concerted effort was made to give the induction a clear structure that could be reproducible for other specialties and for successive rotations of junior doctors. ${ }^{15}$

\section{Reorganization of the ward round and other opportunistic teaching/learning}

The daily ward round is universally recognized to be a good time for opportunistic teaching and learning. However, junior doctors are not presently getting the most out of it. Some readjustments are needed to improve its educational impact. Suggestions in the literature include involvement of 
all team members to sustain everyone's interest, while maintaining adequate control so that teaching points can be made. Additionally, the order of patients seen on the round should be planned, so that both clinical issues and teaching points are sufficiently addressed before time pressure becomes a problem. ${ }^{16}$ The teaching should be in a structured manner, and learning points should be made explicit. ${ }^{17}$

Before starting the round, it may be beneficial to ask the juniors their learning objectives, for example, what they want to focus on or gain from the ward round. This will facilitate their actual learning needs and enable them to undertake focused self-directed study upon completion of the ward round to consolidate the knowledge/concepts acquired. To apply these principles to real-life clinical practice, junior doctors may be given the responsibility of having their own patient to clerk before presenting to their consultant during the ward round. They can then discuss the rationale for investigations and treatment and receive on-the-spot teaching and feedback related to the cases in which they are directly involved in. With regard to concerns about extra time consumption and inefficiency if teaching was to happen during ward rounds, a proposed solution is to give junior doctor(s) the responsibility of one patient each, while the senior doctor(s) review other patients and join their junior colleagues afterward.

Junior doctors may also find it helpful to have opportunistic learning relevant to their daily work in the clinical environment, such as in outpatient clinics or the operating theater. With the patient case readily presented in front of them, the context and learning points will be clear and easily understood.

The process is beneficial to both the learner and the trainer on a daily basis. For the trainees, this provides both valuable learning and job satisfaction. From the senior doctors' perspectives, they often find teaching juniors in the clinical setting enjoyable. They believe that this not only fulfills their role as mentors but also produces safer, more competent, and knowledgeable junior staff.

\section{Structured medical curriculum with clear learning objectives}

Postgraduate medical curricula, and particularly the foundation program, is an outcome-based curriculum that focuses on the kind of doctor being produced, rather than the actual educational process. ${ }^{18}$ Despite this being the case, the foundation program does encourage junior doctors to set learning objectives and reflect on their progress in partnership with their clinical and/or educational supervisor. They do have set core competencies and some flexibility to allow for variation in different specialties.

In practice, however, junior doctors in neurosurgery unfortunately still feel that their current learning is unstructured with unclear objectives. They would like a curriculum designed by senior and experienced clinicians, as well as approved by their peers/foundation predecessors, who can provide them with some focus on the relevant topics in the specialty. To this effect, a neurosurgical theme of the week can be trialed. This will put other aspects of training (ward rounds, opportunistic learning/teaching, and workplacebased assessments) into context, while ensuring a benefit toward actual clinical practice. As the themes build on previous learning and the trainees gradually increase their knowledge, they will develop more confidence to treat this particular group of patients. On the other hand, as mentioned earlier, understanding and expectations of training vary between the teachers and the learners, so themes and learning objectives need to be made clear and specific from the beginning.

\section{Awareness of medical education in neurosurgery}

Another idea put forward in the literature is raising awareness of medical education and the training of junior doctors to the neurosurgical faculty, or better yet, involving the trainees themselves. This idea stems from the findings that the perception of learning experience being positive or negative depends largely on the working environment, ${ }^{19}$ and junior doctors are more willing to engage in this environment if they have some input in shaping and tailoring it to their needs. ${ }^{20}$ The ultimate aim is to allow junior trainees to have an open forum where they can discuss subjective barriers to learning, suggest changes, and use existing evidence in medical education to work with their senior colleagues to improve learning opportunities.

This specifically enables junior doctors to lead their own learning and senior doctors to center their teaching on the learners. To avoid cutting into clinical time, this forum could be integrated into regular teaching sessions that are already in place in the juniors' timetable. Many junior doctors do not undertake formal postgraduate course in medical education, and increasing the awareness of up to date evidence-based medical education practices and concepts will broaden the academic aspect of their postgraduate training and positively influence their future practice. 


\section{Other suggestions for improvement of junior doctors' learning}

Another consideration for practice is encouraging more peer-led teaching. Evidence suggests that peer-led teaching and learning is highly effective; ${ }^{21}$ therefore, it may be better for the incoming junior doctors to receive induction and/or training of their jobs from the outgoing junior doctors rather than the senior clinicians who actually in practice do not do the task themselves.

Medical simulation has been proven to be effective at not only teaching procedural skills, teamwork and communication, but also in enhancing the learning of basic sciences and clinical knowledge. ${ }^{22}$ Due to the disjunction between learning in the classroom and in the clinical setting, simulators are valuable learning tools in medical training, particularly if they can be used on demand to help junior doctors fill in the gaps in their own clinical skill set. Used along traditional teaching tools, simulators can enhance the productivity and variation in workplace-based learning. ${ }^{23-25}$

\section{Discussion}

There have been attempts to change workplace learning by senior clinicians; however, there are a number of reasons why these may not be well received by junior doctors, and therefore, not followed through. It could be due to a lack of engagement by junior doctors, complex working patterns, high clinical workload or pressures, or the job in general. Consequently, trainees suffer from low learning motivation and job satisfaction. Neurosurgery is now less competitive at the point of entry into specialty training than before. This is a direct result from fewer applicants - competition ratios have reduced from 15.9 applicants per place in 2012 to 6.6 applicants per place in 2014 (HEE, 2015). ${ }^{26}$ If learning experiences were enhanced for junior doctors during a 4-month rotation as a foundation doctor, it may potentially encourage more applicants to this specialty.

Transitions as a junior doctor not only occur when one qualifies but also are the on-going processes that can be difficult to adapt at the beginning of each rotation in a new department. ${ }^{27}$ It has been well documented in the literature that support and education by institutions are poor for junior doctors as they first enter the clinical practice.$^{28}$ How integrated junior doctors feel in their teams is closely linked to their perception of the workplace, job satisfaction, and learning experience. Consequently, they thrive when given responsibility, as this makes them feel their role is worthwhile and enhances their learning through asking questions, seek- ing guidance, and continually applying knowledge to clinical practice. This needs to be specifically encouraged in newly qualified doctors, since a recurrent theme related to learning in the workplace is that junior doctors do not engage in learning opportunities unless they are made explicit. Trainees need to be motivated to advance from observing in the periphery, in which they are used to as medical students, to full participation and engagement as a member of the clinical team. This might involve them being given an identity and membership to join the community. ${ }^{29}$

To be able to effectively communicate and become an integrated member, it is crucial to learn the language of the community in which one is practicing in. ${ }^{29}$ The neurosurgical ward operates on many jargons and abbreviations in which most junior doctors will be unfamiliar with unless they have rotated through the department previously. As this has a direct impact on their knowledge and patient care, it would be helpful to develop an intervention to address this. This could be addressed through a booklet or presentation during the induction period or before their rotation in the specialty, or alternatively, through peer-led teaching while working in the department.

To sustain a successful community of practice, all members need to be clear as to what their roles and responsibilities are, and how they fit into the daily running of the team. A frequent problem faced at present in neurosurgery is the lack of clear direction for junior doctors due to different daily workloads and frequent clinical priorities. As with learning opportunities, specific roles and responsibilities need to be delegated to each trainee to facilitate effective management of the workload and opportunistic learning.

These ideas for interventions need to be taken forward from theory to application in the actual clinical environment to see whether they make a difference to learning experience of neurosurgical trainees. They will need to be continuously reviewed and adjusted to adapt to the settings they are being applied to - these do not only include the neurosurgical ward but also other specialties, and throughout one's clinical career.

\section{Limitations}

This paper highlights various generic concerns and issues relating to teaching and training in neurosurgery for junior doctors. There are several barriers that exist to learning, which relates to training/learning dissatisfaction, that have not been addressed. Further work needs to be done specifically in neurosurgery to address on a larger scale, as opposed to one unit in the UK, the real reasons junior doctors feel 
dissatisfied and what actually can be done to improve training and learning satisfaction on a larger scale.

It would also be useful to assess specifically what the current teaching is effective at helping with in terms of confidence, competence, and workplace satisfaction when related to training. This could be done on a large-scale basis, and the collected data were analyzed to find the specific root causes for problems with training and options for improving this to enhance training satisfaction.

\section{Conclusion}

The initial transition from medical student to junior doctor following qualification is a universally exciting and challenging period. The entailed changes have an effect on many aspects of clinical practice among junior doctors, one of which is learning in the workplace through self-directed learning or on-the-job learning. Learning clearly continues from qualification throughout one's career; however, there are specific barriers during this initial period that lead to suboptimal learning experiences and, consequently, satisfaction in trainees. These include feeling underprepared for their new jobs even after an induction period, low job morale as they do not feel well integrated in the team, and a lack of clear learning directions. Despite the fact that both senior and junior doctors appreciate the importance of teaching/learning in the workplace, they are too pressurized by the workload to make time for structured teaching, especially in the neurosurgical department where there are frequent clinical emergencies.

Suggested interventions include adjustments in the ward rounds to become more learner focused, as well as changes in the induction program and a specialty relevant curriculum. We consider ways of integrating junior doctors into the team to improve their perception of the workplace, job satisfaction, and learning experience. An open forum for discussion of medical education between junior and senior doctors is encouraged to establish communication and mutual understanding of teaching/learning in the workplace.

\section{Disclosure}

The authors report no conflicts of interest in this work.

\section{References}

1. Billett $\mathrm{S}$. Workplace pedagogic practices: co-participation and learning. Br J Educ Stud. 2002;50(4):457-481.

2. Goodfellow PB, Claydon P. Students sitting medical finals - ready to be house officers? J R Soc Med. 2001;94(10):516-520.

3. Moercke AM, Eika B. What are the clinical skills levels of newly graduated physicians? Self-assessment study of an intended curriculum identified by a Delphi process. Med Educ. 2002;36(5):472-478.
4. Burch VC, Nash RC, Zabow T, et al. A structured assessment of newly qualified medical graduates. Med Educ. 2005;39(7):723-731.

5. Berridge EJ, Freeth D, Sharpe J, Roberts CM. Bridging the gap: supporting the transition from medical student to practising doctor - a two-week preparation programme after graduation. Med Teach. 2007;29(2-3):119-127.

6. Evans DE, Wood DF, Roberts CM. The effect of an extended hospital induction on perceived confidence and assessed clinical skills of newly qualified pre-registration house officers. Med Educ. 2004:38(9):998-1001.

7. Brown J, Chapman T, Graham D. Becoming a new doctor: a learning or survival exercise? Med Educ. 2007;41(7):653-660.

8. Miles S, Kellett J, Leinster SJ. Foundation doctors' induction experiences. BMC Med Educ. 2015;15:118.

9. Billett S. Learning through work: workplace affordances and individual engagement. J Work Learn. 2001;13(5):209-214.

10. Goodyear HM. First year doctors experience of work related wellbeing and implications for educational provision. Int J Med Educ. 2014;5:103-109.

11. Qureshi NS, Swamy NN. Postgraduate trainees' assessment of the educational value of ward rounds in obstetrics and gynaecology. $J$ Obstet Gynaecol. 2008;28(7):671-675.

12. Harden RM. What is a spiral curriculum? Med Teach. 1999;21(2): 141-143.

13. Cottingham AH, Suchman AL, Litzelman DK, et al. Enhancing the informal curriculum of a medical school: a case study in organizational culture change. J Gen Intern Med. 2008;23(6):715-722.

14. Swanwick T. Informal learning in postgraduate medical education: from cognitivism to "culturism". Med Educ. 2005;39(8):859-865.

15. Acharya V, Mansour S, Amis SM, Reyahi A. Can the transition process from foundation doctor to neurosurgical specialty trainee be improved through "learner-centered induction programs"? Adv Med Educ Pract. 2015;6:591-595.

16. Walton JM, Steinert Y. Patterns of interaction during rounds: implications for work-based learning. Med Educ. 2010;44(6):550-558

17. Stanley P. Structuring ward rounds for learning: can opportunities be created? Med Educ. 1998;32(3):239-243.

18. Harden RM, Crosby JR, Davis MH. AMEE guide no. 14: outcomebased education: part 1-an introduction to outcome-based education. Med Teach. 1999;21(1):7-14.

19. Boor K, Scheele F, van der Vleuten CPM, Teunissen PW, den Breejen EM, Scherpbier AJ. How undergraduate clinical learning climates differ: a multi-method case study. Med Educ. 2008;42(10): 1029-1036.

20. Gray M, Aspland T. Midwifery practice in the university context: perspectives of postgraduate students on the effectiveness of casebased learning in preparation for the workplace. Teach Learn Nurs. 2011;6(2):38-45.

21. Buckley S, Zamora J. Effects of participation in a cross year peer tutoring programme in clinical examination skills on volunteer tutors' skills and attitudes towards teachers and teaching. BMC Med Educ. 2007;7:20.

22. Bradley P. The history of simulation in medical education and possible future directions. Med Educ. 2006;40(3):254-262.

23. Gordon JA, Pawlowski J. Education on-demand: the development of a simulator-based medical education service. Acad Med. 2002; 77(7):751-752.

24. Khan K, Pattison T, Sherwood M. Simulation in medical education. Med Teach. 2011;33(1):1-3.

25. Okuda Y, Bryson EO, DeMaria S, et al. The utility of simulation in medical education: what is the evidence? Mt Sinai J Med. 2009; 76(4):330-343.

26. Health Education England [webpage on the Internet]. 2014 Competition Ratios. Available from: http://specialtytraining.hee.nhs.uk/specialtyrecruitment/competition-ratios/2014-competition-ratios/. Accessed January 20, 2016 
27. Kilminster S, Zukas M, Quinton N, Roberts T. Preparedness is not enough: understanding transitions as critically intensive learning periods. Med Educ. 2011;45(10):1006-1015.

28. Luthy C, Perrier A, Perrin E, Cedraschi C, Allaz AF. Exploring the major difficulties perceived by residents in training: a pilot study. Swiss Med Wkly. 2004;134(41-42):612-617.
29. Lave J, Wenger E. Situated Learning: Legitimate Peripheral Participation. Cambridge: Cambridge University Press; 1991.

\section{Publish your work in this journal}

Advances in Medical Education and Practice is an international, peerreviewed, open access journal that aims to present and publish research on Medical Education covering medical, dental, nursing and allied health care professional education. The journal covers undergraduate education, postgraduate training and continuing medical education including emerging trends and innovative models linking education, research, and health care services. The manuscript management system is completely online and includes a very quick and fair peer-review system. Visit http://www.dovepress.com/testimonials.php to read real quotes from published authors.

Submit your manuscript here: http://www.dovepress.com/advances-in-medical-education-and-practice-journal 